\title{
Some convolution identities for Frobenius-Euler polynomials
}

Jing Pan and Fengzao Yang*

\section{"Correspondence: \\ yangfengzao@126.com \\ Department of Mathematics, \\ Kunming University of Science and \\ Technology, Kunming, Yunnan \\ 650500 , People's Republic of China}

\begin{abstract}
In this paper, by applying the generating function methods and summation transform techniques, we establish some new convolution identities for the Frobenius-Euler polynomials. It turns out that some well-known results are obtained as special cases.
\end{abstract}

MSC: 11B68; 11S40; 05A19

Keywords: Frobenius-Euler polynomials and numbers; Euler polynomials and numbers; Bernoulli polynomials and numbers; combinatorial identities

\section{Introduction}

The classical Frobenius-Euler polynomials $H_{n}(x \mid \lambda)$ are usually defined by the generating function:

$$
\begin{aligned}
& \frac{1-\lambda}{e^{t}-\lambda} e^{x t}=\sum_{n=0}^{\infty} H_{n}(x \mid \lambda) \frac{t^{n}}{n !} \\
& \quad(|t|<\pi \text { if } \lambda=-1 ;|t|<\log (1 / \lambda) \text { otherwise }) .
\end{aligned}
$$

In particular, the case $x=0$ in (1.1) is called the classical Frobenius-Euler numbers given by $H_{n}(\lambda)=H_{n}(0 \mid \lambda)$. It is worthy of mentioning that the classical Frobenius-Euler polynomials and numbers was firstly introduced and studied in great detail by Frobenius [1]. We also refer to [2-10] for some interesting properties on the classical Frobenius-Euler polynomials and numbers.

The widely investigated analogs of the classical Frobenius-Euler polynomials are the classical Bernoulli polynomials $B_{n}(x)$ and the classical Euler polynomials $E_{n}(x)$, which are usually defined by the generating functions (see, e.g., [11-13]):

$$
\begin{aligned}
\frac{t e^{x t}}{e^{t}-1} & =\sum_{n=0}^{\infty} B_{n}(x) \frac{t^{n}}{n !} \quad(|t|<2 \pi), \\
\frac{2 e^{x t}}{e^{t}+1} & =\sum_{n=0}^{\infty} E_{n}(x) \frac{t^{n}}{n !} \quad(|t|<\pi) .
\end{aligned}
$$

The rational numbers $B_{n}$ and integers $E_{n}$ given by

$$
B_{n}=B_{n}(0), \quad E_{n}=2^{n} E_{n}(1 / 2),
$$

(c) The Author(s) 2017. This article is distributed under the terms of the Creative Commons Attribution 4.0 International License (http://creativecommons.org/licenses/by/4.0/), which permits unrestricted use, distribution, and reproduction in any medium, provided you give appropriate credit to the original author(s) and the source, provide a link to the Creative Commons license, and indicate if changes were made. 
are called the classical Bernoulli numbers and the classical Euler numbers, respectively. Obviously, the case $\lambda=-1$ in (1.1) gives the classical Euler polynomials. In fact, the classical Bernoulli polynomials can also be expressed by the classical Frobenius-Euler numbers, as follows:

$$
m^{n-1} \sum_{i=0}^{m-1} \lambda^{i} B_{n}\left(\frac{i}{m}\right)=\frac{n}{\lambda-1} H_{n-1}\left(\frac{1}{\lambda}\right) \quad(m, n \geq 1 ; \lambda \neq 1) .
$$

In the year 2007, Agoh and Dilcher [14] made use of some connections between the classical Bernoulli numbers and the Stirling numbers of the second kind to extend Euler's well-known recurrence formula on the classical Bernoulli numbers:

$$
\sum_{i=0}^{n}\left(\begin{array}{l}
n \\
i
\end{array}\right) B_{i} B_{n-i}=-n B_{n-1}-(n-1) B_{n} \quad(n \geq 1)
$$

and they obtained a convolution identity on the classical Bernoulli numbers, as follows:

$$
\begin{aligned}
\sum_{i=0}^{n}\left(\begin{array}{c}
n \\
i
\end{array}\right) B_{m+n-i} B_{k+i} \\
=-\frac{k ! \cdot m ! \cdot(n+\delta(k, m)(k+m+1))}{(k+m+1) !} B_{k+m+n} \\
\quad+\sum_{i=1}^{k+m}(-1)^{i} \frac{B_{k+m+1-i}}{k+m+1-i}\left\{(-1)^{k}\left(\begin{array}{c}
k+1 \\
i
\end{array}\right)\left(\frac{k+1-i}{k+1} n-\frac{i}{k+1} m\right)\right. \\
\left.\quad+(-1)^{m}\left(\begin{array}{c}
m+1 \\
i
\end{array}\right)\left(\frac{m+1-i}{m+1} n-\frac{i}{m+1} k\right)\right\} B_{n+i-1},
\end{aligned}
$$

where $k, m, n$ are non-negative integers, $\delta(k, m)=0$ when $k=0$ or $m=0$, and $\delta(k, m)=1$ otherwise. Interest in (1.6) stems from its good value distributions, some authors reproved the above formula by applying different methods; see, for example [15-20].

Motivated and inspired by the work of the above authors, in this paper we establish some similar convolution identities for the classical Frobenius-Euler polynomials to (1.6) by applying the generating function methods and summation transform techniques developed in [18]. Accordingly we present some special cases as well as immediate consequences of the main results.

This paper is organized as follows. In the second section, we state some new convolution identities for the classical Frobenius-Euler polynomials, by virtue of which some known results including the classical ones due to Carlitz [21] are obtained as special cases. In the third and fourth sections are contributions to the proofs of the new convolution identities for the classical Frobenius-Euler polynomials.

\section{The statement of results}

In this section, we shall present some new convolution identities for the classical Frobenius-Euler polynomials, and show some illustrative special cases as well as immediate consequences of the main results. We first state the following results. 
Theorem 2.1 Let $m, n, k$ be non-negative integers. Then, for $\lambda \neq 0,1, \mu \neq 0,1$ and $\lambda \mu \neq 1$,

$$
\begin{aligned}
& \sum_{i=0}^{n}\left(\begin{array}{c}
n \\
i
\end{array}\right) H_{m+n-i}\left(y \mid \frac{1}{\lambda \mu}\right) H_{k+i}\left(x \mid \frac{1}{\mu}\right) \\
&=\frac{\lambda \mu-1}{\lambda-1} \sum_{i=0}^{k}\left(\begin{array}{c}
k \\
i
\end{array}\right)(-1)^{k-i} H_{m+k-i}\left(y \mid \frac{1}{\lambda}\right) H_{n+i}\left(x+y \mid \frac{1}{\mu}\right) \\
&-\frac{\lambda(\mu-1)}{\lambda-1} \sum_{i=0}^{m}\left(\begin{array}{c}
m \\
i
\end{array}\right)(-1)^{m-i} H_{m+k-i}(x \mid \lambda) H_{n+i}\left(x+y \mid \frac{1}{\lambda \mu}\right) .
\end{aligned}
$$

It follows that we give some special cases of Theorem 2.1. Since the classical FrobeniusEuler polynomials obey the symmetric distributions (see, e.g., [22])

$$
H_{n}(1-x \mid \lambda)=(-1)^{n} H_{n}\left(x \mid \frac{1}{\lambda}\right) \quad(n \geq 0 ; \lambda \neq 0,1)
$$

by setting $x+y=1-z$ in Theorem 2.1 , we get the following result.

Corollary 2.2 Let $m, n, k$ be non-negative integers. Then, for $\lambda \neq 0,1, \mu \neq 0,1, \lambda \mu \neq 1$, and $x+y+z=1$,

$$
\begin{aligned}
& (-1)^{n}(\lambda-1) \sum_{i=0}^{n}\left(\begin{array}{c}
n \\
i
\end{array}\right) H_{m+n-i}\left(y \mid \frac{1}{\lambda \mu}\right) H_{k+i}\left(x \mid \frac{1}{\mu}\right) \\
& +(-1)^{k}(1-\lambda \mu) \sum_{i=0}^{k}\left(\begin{array}{c}
k \\
i
\end{array}\right) H_{m+k-i}\left(y \mid \frac{1}{\lambda}\right) H_{n+i}(z \mid \mu) \\
& +(-1)^{m} \lambda(\mu-1) \sum_{i=0}^{m}\left(\begin{array}{c}
m \\
i
\end{array}\right) H_{m+k-i}(x \mid \lambda) H_{n+i}(z \mid \lambda \mu)=0 .
\end{aligned}
$$

If we take $k=0$ in Theorem 2.1, we get for non-negative integers $m, n$, and $\lambda \neq 0,1$, $\mu \neq 0,1, \lambda \mu \neq 1$,

$$
\begin{aligned}
H_{m}\left(y \mid \frac{1}{\lambda}\right) H_{n}\left(x+y \mid \frac{1}{\mu}\right)= & \frac{\lambda(\mu-1)}{\lambda \mu-1} \sum_{i=0}^{m}\left(\begin{array}{c}
m \\
i
\end{array}\right)(-1)^{m-i} H_{m-i}(x \mid \lambda) H_{n+i}\left(x+y \mid \frac{1}{\lambda \mu}\right) \\
& +\frac{\lambda-1}{\lambda \mu-1} \sum_{i=0}^{n}\left(\begin{array}{c}
n \\
i
\end{array}\right) H_{m+i}\left(y \mid \frac{1}{\lambda \mu}\right) H_{n-i}\left(x \mid \frac{1}{\mu}\right) .
\end{aligned}
$$

By substituting $x$ for $y, y$ for $x+y, \lambda$ for $1 / \lambda$, and $\mu$ for $1 / \mu$ in (2.4), we have

$$
\begin{aligned}
H_{m}(x \mid \lambda) H_{n}(y \mid \mu)= & \frac{\mu-1}{\lambda \mu-1} \sum_{i=0}^{m}\left(\begin{array}{c}
m \\
i
\end{array}\right)(-1)^{m-i} H_{m-i}\left(y-x \mid \frac{1}{\lambda}\right) H_{n+i}(y \mid \lambda \mu) \\
& +\frac{\mu(\lambda-1)}{\lambda \mu-1} \sum_{i=0}^{n}\left(\begin{array}{c}
n \\
i
\end{array}\right) H_{m+i}(x \mid \lambda \mu) H_{n-i}(y-x \mid \mu) .
\end{aligned}
$$


Since the classical Frobenius-Euler polynomials satisfy the difference equation (see, e.g., [22])

$$
H_{n}(1+x \mid \lambda)-\lambda H_{n}(x \mid \lambda)=(1-\lambda) x^{n} \quad(n \geq 0 ; \lambda \neq 1),
$$

from (2.2) and (2.6), we obtain

$$
(-1)^{n} H_{n}\left(x \mid \frac{1}{\lambda}\right)=(1-\lambda)(-x)^{n}+\lambda H_{n}(-x \mid \lambda) \quad(n \geq 0 ; \lambda \neq 0,1) .
$$

Hence, by applying (2.7) to (2.5), we get the following formula for the products of the classical Frobenius-Euler polynomials.

Corollary 2.3 Let $m, n$ be non-negative integers. Then, for $\lambda \neq 1, \mu \neq 1$, and $\lambda \mu \neq 1$,

$$
\begin{aligned}
& H_{m}(x \mid \lambda) H_{n}(y \mid \mu) \\
& =\frac{\lambda(\mu-1)}{\lambda \mu-1} \sum_{i=0}^{m}\left(\begin{array}{c}
m \\
i
\end{array}\right) H_{m-i}(x-y \mid \lambda) H_{n+i}(y \mid \lambda \mu) \\
& +\frac{\mu(\lambda-1)}{\lambda \mu-1} \sum_{i=0}^{n}\left(\begin{array}{c}
n \\
i
\end{array}\right) H_{n-i}(y-x \mid \mu) H_{m+i}(x \mid \lambda \mu) \\
& \quad-\frac{(\lambda-1)(\mu-1)}{\lambda \mu-1} \sum_{i=0}^{m}\left(\begin{array}{c}
m \\
i
\end{array}\right)(x-y)^{m-i} H_{n+i}(y \mid \lambda \mu) .
\end{aligned}
$$

In particular, the case $x=y$ in Corollary 2.3 gives for non-negative integers $m, n$ and $\lambda \neq 1, \mu \neq 1, \lambda \mu \neq 1$,

$$
\begin{aligned}
& H_{m}(x \mid \lambda) H_{n}(x \mid \mu) \\
& =\frac{\lambda(\mu-1)}{\lambda \mu-1} \sum_{i=0}^{m}\left(\begin{array}{c}
m \\
i
\end{array}\right) H_{i}(\lambda) H_{m+n-i}(x \mid \lambda \mu) \\
& \quad+\frac{\mu(\lambda-1)}{\lambda \mu-1} \sum_{i=0}^{n}\left(\begin{array}{c}
n \\
i
\end{array}\right) H_{i}(\mu) H_{m+n-i}(x \mid \lambda \mu) \\
& \quad-\frac{(\lambda-1)(\mu-1)}{\lambda \mu-1} H_{m+n}(x \mid \lambda \mu),
\end{aligned}
$$

which was firstly discovered by Carlitz [21]. Corollary 2.3 can also be found in [23], Theorem 2.1, where it was used to give, for non-negative integer $n$ and $\lambda \neq \pm 1$,

$$
\begin{aligned}
& \sum_{i=0}^{n} H_{i}(x \mid \lambda) H_{n-i}(x \mid \lambda) \\
& \quad=\frac{2 \lambda}{\lambda+1} \sum_{i=0}^{n}\left(\begin{array}{c}
n+1 \\
i
\end{array}\right) H_{n-i}(\lambda) H_{i}\left(x \mid \lambda^{2}\right)-\frac{\lambda-1}{\lambda+1}(n+1) H_{n}\left(x \mid \lambda^{2}\right),
\end{aligned}
$$


and for positive integer $n \geq 2$ and $\lambda \neq \pm 1$,

$$
\begin{aligned}
& \sum_{i=1}^{n-1} \frac{H_{i}(x \mid \lambda) H_{n-i}(x \mid \lambda)}{i(n-i)} \\
& \quad=\frac{\lambda}{\lambda+1} \cdot \frac{2}{n} \sum_{i=1}^{n-1}\left(\begin{array}{c}
n \\
i
\end{array}\right)\left(H_{n-1}-H_{i-1}\right) H_{i}(\lambda) H_{n-i}\left(x \mid \lambda^{2}\right)+2 H_{n-1} \frac{H_{n}\left(x \mid \lambda^{2}\right)}{n},
\end{aligned}
$$

where $H_{n}$ is the Harmonic numbers given by

$$
H_{0}=1 \quad \text { and } \quad H_{n}=\sum_{i=1}^{n} \frac{1}{i}=1+\frac{1}{2}+\cdots+\frac{1}{n} \quad(n \geq 1) .
$$

Equations (2.10) and (2.11) are very analogous to the following convolution identities on the classical Bernoulli polynomials due to Kim et al. [24], namely

$$
\sum_{i=0}^{n} B_{i}(x) B_{n-i}(x)=\frac{2}{n+2} \sum_{i=0}^{n-2}\left(\begin{array}{c}
n+2 \\
i
\end{array}\right) B_{n-i} B_{i}(x)+(n+1) B_{n}(x) \quad(n \geq 2)
$$

and

$$
\sum_{i=1}^{n-1} \frac{B_{i}(x) B_{n-i}(x)}{i(n-i)}=\frac{2}{n} \sum_{i=0}^{n-2}\left(\begin{array}{c}
n \\
i
\end{array}\right) \frac{B_{n-i} B_{i}(x)}{n-i}+\frac{2}{n} H_{n-1} B_{n}(x) \quad(n \geq 2) .
$$

The case $x=0$ in (2.13) and (2.14) will lead to the famous Miki identity and the famous Matiyasevich identity on the classical Bernoulli numbers, respectively. For some related results of (2.10), (2.11), (2.13), and (2.14), one may consult [22, 25-29].

Theorem 2.4 Let $m, n, k$ be non-negative integers. Then, for $\lambda \neq 0,1$,

$$
\begin{aligned}
\sum_{i=0}^{n}\left(\begin{array}{c}
n \\
i
\end{array}\right) \beta_{m+n-i}(x) H_{k+i}(y \mid \lambda) \\
=\frac{1}{\lambda-1} \sum_{i=0}^{k}\left(\begin{array}{c}
k \\
i
\end{array}\right)(-1)^{k-i} H_{m+k-i}\left(x \mid \frac{1}{\lambda}\right) H_{n+i}(x+y \mid \lambda) \\
\quad+\sum_{i=0}^{m}\left(\begin{array}{c}
m \\
i
\end{array}\right)(-1)^{m-i} H_{m+k-i}(y \mid \lambda) \beta_{n+i}(x+y) \\
\quad-(-1)^{m} \frac{m ! \cdot n !}{(m+n+1) !} H_{m+n+k+1}(y \mid \lambda),
\end{aligned}
$$

where $\beta_{n}(x)$ is denoted by $\beta_{n}(x)=B_{n+1}(x) /(n+1)$ for non-negative integer $n$.

It is clear that the classical Bernoulli polynomials satisfy the symmetric distributions (see, e.g., [30]):

$$
B_{n}(1-x)=(-1)^{n} B_{n}(x) \quad(n \geq 0)
$$


which means

$$
\beta_{n}(1-x)=(-1)^{n+1} \beta_{n}(x) \quad(n \geq 0) .
$$

Hence, by setting $x+y=1-z$ in Theorem 2.4, in view of (2.2) and (2.17), we get the following result.

Corollary 2.5 Let $m, n, k$ be non-negative integers. Then, for $\lambda \neq 0,1$ and $x+y+z=1$,

$$
\begin{aligned}
& (-1)^{n} \sum_{i=0}^{n}\left(\begin{array}{c}
n \\
i
\end{array}\right) \beta_{m+n-i}(x) H_{k+i}(y \mid \lambda)+(-1)^{k} \frac{1}{1-\lambda} \sum_{i=0}^{k}\left(\begin{array}{c}
k \\
i
\end{array}\right) H_{m+k-i}\left(x \mid \frac{1}{\lambda}\right) H_{n+i}\left(z \mid \frac{1}{\lambda}\right) \\
& \quad+(-1)^{m} \sum_{i=0}^{m}\left(\begin{array}{c}
m \\
i
\end{array}\right) H_{m+k-i}(y \mid \lambda) \beta_{n+i}(z) \\
& =(-1)^{m+n+1} \frac{m ! \cdot n !}{(m+n+1) !} H_{m+n+k+1}(y \mid \lambda),
\end{aligned}
$$

where $\beta_{n}(x)$ is denoted by $\beta_{n}(x)=B_{n+1}(x) /(n+1)$ for non-negative integer $n$.

If we take $\lambda=-1$ in Corollary 2.5, we obtain the following convolution identity for the classical Euler polynomials.

Corollary 2.6 Let $m, n, k$ be non-negative integers. Then, for $x+y+z=1$,

$$
\begin{aligned}
& (-1)^{n} \sum_{i=0}^{n}\left(\begin{array}{c}
n \\
i
\end{array}\right) \beta_{m+n-i}(x) E_{k+i}(y)+(-1)^{k} \frac{1}{2} \sum_{i=0}^{k}\left(\begin{array}{c}
k \\
i
\end{array}\right) E_{m+k-i}(x) E_{n+i}(z) \\
& +(-1)^{m} \sum_{i=0}^{m}\left(\begin{array}{c}
m \\
i
\end{array}\right) E_{m+k-i}(y) \beta_{n+i}(z) \\
& =(-1)^{m+n+1} \frac{m ! \cdot n !}{(m+n+1) !} E_{m+n+k+1}(y),
\end{aligned}
$$

where $\beta_{n}(x)$ is denoted by $\beta_{n}(x)=B_{n+1}(x) /(n+1)$ for non-negative integer $n$.

If we substitute $y-x$ for $y$ in Theorem 2.4, we get for non-negative integers $m, n, k$ and $\lambda \neq 0,1$,

$$
\begin{aligned}
\sum_{i=0}^{n}\left(\begin{array}{c}
n \\
i
\end{array}\right) \beta_{m+n-i}(x) H_{k+i}(y-x \mid \lambda) \\
=\frac{1}{\lambda-1} \sum_{i=0}^{k}\left(\begin{array}{c}
k \\
i
\end{array}\right)(-1)^{k-i} H_{m+k-i}\left(x \mid \frac{1}{\lambda}\right) H_{n+i}(y \mid \lambda) \\
\quad+\sum_{i=0}^{m}\left(\begin{array}{c}
m \\
i
\end{array}\right)(-1)^{m-i} H_{m+k-i}(y-x \mid \lambda) \beta_{n+i}(y) \\
\quad-(-1)^{m} \frac{m ! \cdot n !}{(m+n+1) !} H_{m+n+k+1}(y-x \mid \lambda) .
\end{aligned}
$$


By setting $m=0$ in (2.20), we get for non-negative integers $n, k$ and $\lambda \neq 0,1$,

$$
\begin{aligned}
& \beta_{n}(y) H_{k}(y-x \mid \lambda) \\
& =\sum_{i=0}^{n}\left(\begin{array}{c}
n \\
i
\end{array}\right) \beta_{n-i}(x) H_{k+i}(y-x \mid \lambda) \\
& \quad-\frac{1}{\lambda-1} \sum_{i=0}^{k}\left(\begin{array}{c}
k \\
i
\end{array}\right)(-1)^{k-i} H_{k-i}\left(x \mid \frac{1}{\lambda}\right) H_{n+i}(y \mid \lambda)+\frac{1}{n+1} H_{n+k+1}(y-x \mid \lambda) .
\end{aligned}
$$

Substituting $x$ for $y, x-y$ for $x, m$ for $n$, and $n$ for $k$ in (2.21) gives

$$
\begin{aligned}
& \beta_{m}(x) H_{n}(y \mid \lambda) \\
& =\sum_{i=0}^{m}\left(\begin{array}{c}
m \\
i
\end{array}\right) \beta_{m-i}(x-y) H_{n+i}(y \mid \lambda) \\
& \quad-\frac{1}{\lambda-1} \sum_{i=0}^{n}\left(\begin{array}{c}
n \\
i
\end{array}\right)(-1)^{n-i} H_{n-i}\left(x-y \mid \frac{1}{\lambda}\right) H_{m+i}(x \mid \lambda)+\frac{1}{m+1} H_{m+n+1}(y \mid \lambda) .
\end{aligned}
$$

It follows from (2.7) and (2.22) that, for non-negative integers $m, n$ and $\lambda \neq 1$,

$$
\begin{aligned}
B_{m+1}(x) H_{n}(y \mid \lambda)= & \sum_{i=0}^{m+1}\left(\begin{array}{c}
m+1 \\
i
\end{array}\right) B_{m+1-i}(x-y) H_{n+i}(y \mid \lambda) \\
& -\frac{\lambda}{\lambda-1}(m+1) \sum_{i=0}^{n}\left(\begin{array}{c}
n \\
i
\end{array}\right) H_{n-i}(y-x \mid \lambda) H_{m+i}(x \mid \lambda) \\
& +(m+1) \sum_{i=0}^{n}\left(\begin{array}{c}
n \\
i
\end{array}\right)(y-x)^{n-i} H_{m+i}(x \mid \lambda) .
\end{aligned}
$$

Notice that, for non-negative integers $m, n$ (see, e.g., [31], Theorem 1.1),

$$
\sum_{i=0}^{m}\left(\begin{array}{c}
m \\
i
\end{array}\right) x^{m-i} f_{n+i}(y)=\sum_{i=0}^{n}\left(\begin{array}{c}
n \\
i
\end{array}\right)(-x)^{n-i} f_{m+i}(x+y),
$$

where $f_{n}(x)$ is a sequence of polynomials given for formal power series $F(t)$ by

$$
\sum_{n=0}^{\infty} f_{n}(x) \frac{t^{n}}{n !}=F(t) e^{(x-1 / 2) t}
$$

If we take $F(t)=(1-\lambda) e^{t / 2} /\left(e^{t}-\lambda\right)$ in (2.25) and then substitute $x-y$ for $x$ in (2.24), we obtain for non-negative integers $m, n$ and $\lambda \neq 1$,

$$
\sum_{i=0}^{m}\left(\begin{array}{c}
m \\
i
\end{array}\right)(x-y)^{m-i} H_{n+i}(y \mid \lambda)=\sum_{i=0}^{n}\left(\begin{array}{c}
n \\
i
\end{array}\right)(y-x)^{n-i} H_{m+i}(x \mid \lambda) .
$$

Thus, by applying (2.26) to (2.23), we get the following formula for the products of the classical Bernoulli and Frobenius-Euler polynomials. 
Corollary 2.7 Let $m, n$ non-negative integers. Then, for $\lambda \neq 1$,

$$
\begin{aligned}
B_{m+1}(x) H_{n}(y \mid \lambda)= & \sum_{i=0}^{m+1}\left(\begin{array}{c}
m+1 \\
i
\end{array}\right) B_{m+1-i}(x-y) H_{n+i}(y \mid \lambda) \\
& -\frac{\lambda}{\lambda-1}(m+1) \sum_{i=0}^{n}\left(\begin{array}{c}
n \\
i
\end{array}\right) H_{n-i}(y-x \mid \lambda) H_{m+i}(x \mid \lambda) \\
& +(m+1) \sum_{i=0}^{m}\left(\begin{array}{c}
m \\
i
\end{array}\right)(x-y)^{m-i} H_{n+i}(y \mid \lambda) .
\end{aligned}
$$

If we take $k=0$ in (2.20) then, for non-negative integers $m, n$ and $\lambda \neq 0,1$,

$$
\begin{aligned}
H_{m}\left(x \mid \frac{1}{\lambda}\right) H_{n}(y \mid \lambda)= & -(\lambda-1) \sum_{i=0}^{m}\left(\begin{array}{c}
m \\
i
\end{array}\right)(-1)^{m-i} H_{m-i}(y-x \mid \lambda) \beta_{n+i}(y) \\
& +(\lambda-1) \sum_{i=0}^{n}\left(\begin{array}{c}
n \\
i
\end{array}\right) \beta_{m+i}(x) H_{n-i}(y-x \mid \lambda) \\
& +(\lambda-1) \frac{(-1)^{m} m ! \cdot n !}{(m+n+1) !} H_{m+n+1}(y-x \mid \lambda),
\end{aligned}
$$

which together with (2.7) yields the following result.

Corollary 2.8 Let $m, n$ be non-negative integers. Then, $\lambda \neq 0,1$,

$$
\begin{aligned}
& H_{m}\left(x \mid \frac{1}{\lambda}\right) H_{n}(y \mid \lambda) \\
& =\left(\frac{1}{\lambda}-1\right) \sum_{i=0}^{m}\left(\begin{array}{c}
m \\
i
\end{array}\right) H_{m-i}\left(x-y \mid \frac{1}{\lambda}\right) \frac{B_{n+i+1}(y)}{n+i+1} \\
& +(\lambda-1) \sum_{i=0}^{n}\left(\begin{array}{l}
n \\
i
\end{array}\right) H_{n-i}(y-x \mid \lambda) \frac{B_{m+i+1}(x)}{m+i+1} \\
& -\frac{(\lambda-1)^{2}}{\lambda} \sum_{i=0}^{m}\left(\begin{array}{c}
m \\
i
\end{array}\right)(x-y)^{m-i} \frac{B_{n+i+1}(y)}{n+i+1} \\
& +(\lambda-1) \frac{(-1)^{m} m ! \cdot n !}{(m+n+1) !} H_{m+n+1}(y-x \mid \lambda) .
\end{aligned}
$$

In particular, the case $x=y$ in Corollary 2.7 gives that if $\lambda \neq 1$ then, for positive integer $m$ and non-negative integer $n$,

$$
\begin{aligned}
& B_{m}(x) H_{n}(x \mid \lambda) \\
& =\sum_{i=0}^{m}\left(\begin{array}{c}
m \\
i
\end{array}\right) B_{i} H_{m+n-i}(x \mid \lambda) \\
& \quad-\frac{\lambda}{\lambda-1} m \sum_{i=0}^{n}\left(\begin{array}{c}
n \\
i
\end{array}\right) H_{i}(\lambda) H_{m+n-1-i}(x \mid \lambda)+m H_{m+n-1}(x \mid \lambda),
\end{aligned}
$$


and if we set $x=y$ and substitute $m$ for $n$ and $n$ for $m$ in Corollary 2.8 then, for positive integers $m, n$ and $\lambda \neq 0,1$,

$$
\begin{aligned}
& H_{m}(x \mid \lambda) H_{n}\left(x \mid \frac{1}{\lambda}\right) \\
& =(\lambda-1) \sum_{i=1}^{m}\left(\begin{array}{c}
m \\
i
\end{array}\right) H_{i}(\lambda) \frac{B_{m+n+1-i}(x)}{m+n+1-i}+\left(\frac{1}{\lambda}-1\right) \sum_{i=1}^{n}\left(\begin{array}{c}
n \\
i
\end{array}\right) H_{i}\left(\frac{1}{\lambda}\right) \frac{B_{m+n+1-i}(x)}{m+n+1-i} \\
& \quad+(-1)^{n}(\lambda-1) \frac{m ! \cdot n !}{(m+n+1) !} H_{m+n+1}(\lambda) .
\end{aligned}
$$

Equations (2.30) and (2.31) were firstly discovered by Carlitz [21] who used them to give the expressions of the products of the classical Bernoulli polynomials and the classical Euler polynomials stated in Nielsen's classical book [12]. For different proofs of Corollaries 2.7 and 2.8, see [23] for details. For some related results on the products of the classical Bernoulli and Euler polynomials, one can refer to [32-34].

\section{The proof of Theorem 2.1}

We first prove the following auxiliary result.

Lemma 3.1 Let $m, n, k$ be non-negative integers. Then, for $\lambda \neq 0,1, \mu \neq 0,1$, and $\lambda \mu \neq 1$,

$$
\begin{aligned}
\sum_{j=0}^{k}\left(\begin{array}{c}
k \\
j
\end{array}\right) \sum_{i=0}^{n}\left(\begin{array}{c}
n \\
i
\end{array}\right) H_{i+j}\left(x \mid \frac{1}{\mu}\right) H_{m+n+k-i-j}\left(y \mid \frac{1}{\lambda \mu}\right) \\
=\frac{\lambda \mu-1}{\lambda-1} H_{m}\left(y \mid \frac{1}{\lambda}\right) H_{n+k}\left(x+y \mid \frac{1}{\mu}\right) \\
\quad-\frac{\lambda(\mu-1)}{\lambda-1} \sum_{i=0}^{m}\left(\begin{array}{c}
m \\
i
\end{array}\right)(-1)^{m-i} H_{m-i}(x \mid \lambda) H_{n+k+i}\left(x+y \mid \frac{1}{\lambda \mu}\right) .
\end{aligned}
$$

Proof It is easily seen that

$$
\frac{1}{\lambda e^{u}-1} \cdot \frac{1}{\mu e^{v}-1}=\left(\frac{\lambda e^{u}}{\lambda e^{u}-1}+\frac{1}{\mu e^{v}-1}\right) \frac{1}{\lambda \mu e^{u+v}-1} .
$$

If we multiply both sides of the above identity by $(\mu-1)(\lambda \mu-1) e^{x v+y(u+v)}$, we get

$$
\begin{aligned}
& \frac{\mu-1}{\mu e^{v}-1} e^{x v} \cdot \frac{\lambda \mu-1}{\lambda \mu e^{u+v}-1} e^{y(u+v)} \\
& \quad=\frac{\lambda \mu-1}{\lambda-1} \cdot \frac{\lambda-1}{\lambda e^{u}-1} e^{y u} \cdot \frac{\mu-1}{\mu e^{v}-1} e^{(x+y) v} \\
& \quad-\frac{\lambda(\mu-1)}{\lambda-1} \cdot \frac{\lambda-1}{\lambda e^{u}-1} e^{(1-x) u} \cdot \frac{\lambda \mu-1}{\lambda \mu e^{u+v}-1} e^{(x+y)(u+v)} .
\end{aligned}
$$

By substituting $1 / \lambda$ for $\lambda$ in (1.1), we have

$$
\frac{\lambda-1}{\lambda e^{u}-1} e^{x u}=\sum_{m=0}^{\infty} H_{m}\left(x \mid \frac{1}{\lambda}\right) \frac{u^{m}}{m !} .
$$


More generally, by the Taylor theorem, we discover

$$
\frac{\lambda-1}{\lambda e^{u+v}-1} e^{x(u+v)}=\sum_{m=0}^{\infty} \sum_{n=0}^{\infty} H_{m+n}\left(x \mid \frac{1}{\lambda}\right) \frac{u^{m}}{m !} \cdot \frac{v^{n}}{n !} .
$$

Applying (3.4) and (3.5) to (3.3) gives

$$
\begin{aligned}
\left(\sum_{n=0}^{\infty} H_{n}\left(x \mid \frac{1}{\mu}\right) \frac{v^{n}}{n !}\right)\left(\sum_{m=0}^{\infty} \sum_{n=0}^{\infty} H_{m+n}\left(y \mid \frac{1}{\lambda \mu}\right) \frac{u^{m}}{m !} \cdot \frac{v^{n}}{n !}\right) \\
=\frac{\lambda \mu-1}{\lambda-1}\left(\sum_{m=0}^{\infty} H_{m}\left(y \mid \frac{1}{\lambda}\right) \frac{u^{m}}{m !}\right)\left(\sum_{n=0}^{\infty} H_{n}\left(x+y \mid \frac{1}{\mu}\right) \frac{v^{n}}{n !}\right) \\
\quad-\frac{\lambda(\mu-1)}{\lambda-1}\left(\sum_{m=0}^{\infty} H_{m}\left(1-x \mid \frac{1}{\lambda}\right) \frac{u^{m}}{m !}\right)\left(\sum_{m=0}^{\infty} \sum_{n=0}^{\infty} H_{m+n}\left(x+y \mid \frac{1}{\lambda \mu}\right) \frac{u^{m}}{m !} \cdot \frac{v^{n}}{n !}\right) .
\end{aligned}
$$

If we take $k$ times the derivative for (3.6) with respect to $v$, in view of the Leibniz rule, we obtain

$$
\begin{aligned}
\sum_{j=0}^{k}\left(\begin{array}{l}
k \\
j
\end{array}\right)\left(\sum_{n=0}^{\infty} H_{n+j}\left(x \mid \frac{1}{\mu}\right) \frac{v^{n}}{n !}\right)\left(\sum_{m=0}^{\infty} \sum_{n=0}^{\infty} H_{m+n+k-j}\left(y \mid \frac{1}{\lambda \mu}\right) \frac{u^{m}}{m !} \cdot \frac{v^{n}}{n !}\right) \\
=\frac{\lambda \mu-1}{\lambda-1}\left(\sum_{m=0}^{\infty} H_{m}\left(y \mid \frac{1}{\lambda}\right) \frac{u^{m}}{m !}\right)\left(\sum_{n=0}^{\infty} H_{n+k}\left(x+y \mid \frac{1}{\mu}\right) \frac{v^{n}}{n !}\right) \\
-\frac{\lambda(\mu-1)}{\lambda-1}\left(\sum_{m=0}^{\infty} H_{m}\left(1-x \mid \frac{1}{\lambda}\right) \frac{u^{m}}{m !}\right) \\
\quad \times\left(\sum_{m=0}^{\infty} \sum_{n=0}^{\infty} H_{m+n+k}\left(x+y \mid \frac{1}{\lambda \mu}\right) \frac{u^{m}}{m !} \cdot \frac{v^{n}}{n !}\right),
\end{aligned}
$$

which together with the Cauchy product and (2.2) yields

$$
\begin{aligned}
\sum_{m=0}^{\infty} \sum_{n=0}^{\infty}\left(\sum_{j=0}^{k}\left(\begin{array}{l}
k \\
j
\end{array}\right) \sum_{i=0}^{n}\left(\begin{array}{c}
n \\
i
\end{array}\right) H_{i+j}\left(x \mid \frac{1}{\mu}\right) H_{m+n+k-i-j}\left(y \mid \frac{1}{\lambda \mu}\right)\right) \frac{u^{m}}{m !} \cdot \frac{v^{n}}{n !} \\
=\sum_{m=0}^{\infty} \sum_{n=0}^{\infty}\left(\frac{\lambda \mu-1}{\lambda-1} H_{m}\left(y \mid \frac{1}{\lambda}\right) H_{n+k}\left(x+y \mid \frac{1}{\mu}\right)\right) \frac{u^{m}}{m !} \cdot \frac{v^{n}}{n !} \\
\quad-\sum_{m=0}^{\infty} \sum_{n=0}^{\infty}\left(\frac{\lambda(\mu-1)}{\lambda-1} \sum_{i=0}^{m}\left(\begin{array}{c}
m \\
i
\end{array}\right)(-1)^{m-i} H_{m-i}(x \mid \lambda)\right. \\
\left.\quad \times H_{n+k+i}\left(x+y \mid \frac{1}{\lambda \mu}\right)\right) \frac{u^{m}}{m !} \cdot \frac{v^{n}}{n !} .
\end{aligned}
$$

Thus, comparing the coefficients of $u^{m} \cdot v^{n} / m ! \cdot n !$ in (3.8) gives the desired result.

We now give the detailed proof of Theorem 2.1.

Proof of Theorem 2.1 We shall use induction on $k$ in Lemma 3.1 to prove Theorem 2.1. Clearly, Theorem 2.1 holds trivially when $k=0$ in Lemma 3.1. Now, we assume that The- 
orem 2.1 holds for all positive integers less than $k$. It follows from Lemma 3.1 that, for non-negative integers $m, n$ and positive integer $k$,

$$
\begin{aligned}
\sum_{i=0}^{n}\left(\begin{array}{c}
n \\
i
\end{array}\right) H_{m+n-i}\left(y \mid \frac{1}{\lambda \mu}\right) H_{k+i}\left(x \mid \frac{1}{\mu}\right) & \\
= & \frac{\lambda \mu-1}{\lambda-1} H_{m}\left(y \mid \frac{1}{\lambda}\right) H_{n+k}\left(x+y \mid \frac{1}{\mu}\right) \\
& -\frac{\lambda(\mu-1)}{\lambda-1} \sum_{i=0}^{m+k}\left(\begin{array}{c}
m \\
i-k
\end{array}\right)(-1)^{m+k-i} H_{m+k-i}(x \mid \lambda) H_{n+i}\left(x+y \mid \frac{1}{\lambda \mu}\right) \\
& -\sum_{j=0}^{k-1}\left(\begin{array}{c}
k \\
j
\end{array}\right) \sum_{i=0}^{n}\left(\begin{array}{c}
n \\
i
\end{array}\right) H_{i+j}\left(x \mid \frac{1}{\mu}\right) H_{m+n+k-i-j}\left(y \mid \frac{1}{\lambda \mu}\right) .
\end{aligned}
$$

Since Theorem 2.1 holds for all positive integers less than $k$, we have

$$
\begin{aligned}
\sum_{j=0}^{k-1}\left(\begin{array}{c}
k \\
j
\end{array}\right) \sum_{i=0}^{n}\left(\begin{array}{c}
n \\
i
\end{array}\right) H_{i+j}\left(x \mid \frac{1}{\mu}\right) H_{m+n+k-i-j}\left(y \mid \frac{1}{\lambda \mu}\right) \\
=\frac{\lambda \mu-1}{\lambda-1} \sum_{i=0}^{k-1}(-1)^{i} H_{m+k-i}\left(y \mid \frac{1}{\lambda}\right) H_{n+i}\left(x+y \mid \frac{1}{\mu}\right) \sum_{j=0}^{k-1}(-1)^{j}\left(\begin{array}{c}
k \\
j
\end{array}\right)\left(\begin{array}{l}
j \\
i
\end{array}\right) \\
\quad-\frac{\lambda(\mu-1)}{\lambda-1} \sum_{i=0}^{m+k}(-1)^{m+k-i} H_{m+k-i}(x \mid \lambda) H_{n+i}\left(x+y \mid \frac{1}{\lambda \mu}\right) \\
\quad \times \sum_{j=0}^{k-1}(-1)^{j}\left(\begin{array}{c}
k \\
j
\end{array}\right)\left(\begin{array}{c}
m+k-j \\
i
\end{array}\right) .
\end{aligned}
$$

Notice that, for non-negative integers $m, n, k, i$,

$$
\sum_{j=0}^{k}(-1)^{j}\left(\begin{array}{c}
k \\
j
\end{array}\right)\left(\begin{array}{c}
m+j \\
i
\end{array}\right)=(-1)^{k}\left(\begin{array}{c}
m \\
i-k
\end{array}\right)
$$

by using induction on $k$. It follows from (3.11) that, for non-negative integers $m, n, k, i$ with $k \geq 1$,

$$
\sum_{j=0}^{k-1}(-1)^{j}\left(\begin{array}{l}
k \\
j
\end{array}\right)\left(\begin{array}{l}
j \\
i
\end{array}\right)=(-1)^{k}\left(\begin{array}{c}
0 \\
i-k
\end{array}\right)-(-1)^{k}\left(\begin{array}{l}
k \\
i
\end{array}\right)
$$

and

$$
\sum_{j=0}^{k-1}(-1)^{j}\left(\begin{array}{c}
k \\
j
\end{array}\right)\left(\begin{array}{c}
m+k-j \\
i
\end{array}\right)=\left(\begin{array}{c}
m \\
i-k
\end{array}\right)-(-1)^{k}\left(\begin{array}{c}
m \\
i
\end{array}\right)
$$

Thus, by applying (3.12) and (3.13) to (3.10) and combining with (3.9) we have the desired result. This completes the proof of Theorem 2.1 . 


\section{The proof of Theorem 2.4}

In a similar consideration to Theorem 2.1, we firstly give the following result.

Lemma 4.1 Let $m, n, k$ be non-negative integers. Then, for $\lambda \neq 0,1$,

$$
\begin{aligned}
\sum_{j=0}^{k}\left(\begin{array}{c}
k \\
j
\end{array}\right) \sum_{i=0}^{n}\left(\begin{array}{c}
n \\
i
\end{array}\right) H_{i+j}(y \mid \lambda) \beta_{m+n+k-i-j}(x) \\
=\frac{1}{\lambda-1} H_{m}\left(x \mid \frac{1}{\lambda}\right) H_{n+k}(x+y \mid \lambda)+\sum_{i=0}^{m}\left(\begin{array}{c}
m \\
i
\end{array}\right)(-1)^{m-i} H_{m-i}(y \mid \lambda) \beta_{n+k+i}(x+y) \\
\quad-(-1)^{m} \frac{m ! \cdot(n+k) !}{(m+n+k+1) !} H_{m+n+k+1}(y \mid \lambda) .
\end{aligned}
$$

Proof By substituting $1 / \lambda$ for $\mu$ in (3.2), we have

$$
\frac{1}{\lambda e^{u}-1} \cdot \frac{1}{\frac{1}{\lambda} e^{v}-1}=\frac{\lambda e^{u}}{\lambda e^{u}-1} \cdot \frac{1}{e^{u+v}-1}+\frac{1}{\frac{1}{\lambda} e^{v}-1} \cdot \frac{1}{e^{u+v}-1} .
$$

Multiplying both sides of the above identity by $(\lambda-1)(1 / \lambda-1) e^{x u+y v}$ yields

$$
\begin{aligned}
\frac{\lambda-1}{\lambda e^{u}-1} e^{x u} \cdot \frac{\frac{1}{\lambda}-1}{\frac{1}{\lambda} e^{v}-1} e^{y v}= & \lambda\left(\frac{1}{\lambda}-1\right) \frac{\lambda-1}{\lambda e^{u}-1} e^{(1+x-y) u}\left(\frac{e^{y(u+v)}}{e^{u+v}-1}-\frac{1}{u+v}+\frac{1}{u+v}\right) \\
& +(\lambda-1) \frac{\frac{1}{\lambda}-1}{\frac{1}{\lambda} e^{v}-1} e^{(y-x) v}\left(\frac{e^{x(u+v)}}{e^{u+v}-1}-\frac{1}{u+v}+\frac{1}{u+v}\right) .
\end{aligned}
$$

It is clear from (1.2) that

$$
\frac{e^{x u}}{e^{u}-1}-\frac{1}{u}=\sum_{m=0}^{\infty} \beta_{m}(x) \frac{u^{m}}{m !}
$$

which together with the Taylor theorem gives

$$
\frac{e^{x(u+v)}}{e^{u+v}-1}-\frac{1}{u+v}=\sum_{m=0}^{\infty} \sum_{n=0}^{\infty} \beta_{m+n}(x) \frac{u^{m}}{m !} \cdot \frac{v^{n}}{n !} .
$$

By applying (3.4) and (4.5) to (3.3), with the help of (2.2), we get

$$
\begin{aligned}
& \left(\sum_{m=0}^{\infty} H_{m}\left(x \mid \frac{1}{\lambda}\right) \frac{u^{m}}{m !}\right)\left(\sum_{n=0}^{\infty} H_{n}(y \mid \lambda) \frac{v^{n}}{n !}\right) \\
& =-(\lambda-1)\left(\sum_{m=0}^{\infty}(-1)^{m} H_{m}(y-x \mid \lambda) \frac{u^{m}}{m !}\right)\left(\sum_{m=0}^{\infty} \sum_{n=0}^{\infty} \beta_{m+n}(y) \frac{u^{m}}{m !} \cdot \frac{v^{n}}{n !}\right) \\
& +(\lambda-1)\left(\sum_{n=0}^{\infty} H_{n}(y-x \mid \lambda) \frac{v^{n}}{n !}\right)\left(\sum_{m=0}^{\infty} \sum_{n=0}^{\infty} \beta_{m+n}(x) \frac{u^{m}}{m !} \cdot \frac{v^{n}}{n !}\right)+M,
\end{aligned}
$$

where $M$ is given by

$$
M=\frac{\lambda-1}{u+v}\left(\sum_{n=0}^{\infty} H_{n}(y-x \mid \lambda) \frac{v^{n}}{n !}-\sum_{m=0}^{\infty}(-1)^{m} H_{m}(y-x \mid \lambda) \frac{u^{m}}{m !}\right) .
$$


Observe that

$$
\sum_{m=0}^{\infty} H_{m}(y-x \mid \lambda) \frac{v^{m}}{m !}=\sum_{m=0}^{\infty} \frac{H_{m}(y-x \mid \lambda)}{m !}(u+v-u)^{m}
$$

which together with the binomial theorem means

$$
\begin{aligned}
& \sum_{m=0}^{\infty} H_{m}(y-x \mid \lambda) \frac{v^{m}}{m !} \\
& \quad=\sum_{m=0}^{\infty} \frac{H_{m}(y-x \mid \lambda)}{m !} \sum_{k=1}^{m}\left(\begin{array}{l}
m \\
k
\end{array}\right)(u+v)^{k}(-u)^{m-k}+\sum_{m=0}^{\infty}(-)^{m} H_{m}(y-x \mid \lambda) \frac{u^{m}}{m !} .
\end{aligned}
$$

It follows from (4.7) and (4.9) that

$$
\begin{aligned}
M & =(\lambda-1) \sum_{m=0}^{\infty} \frac{H_{m}(y-x \mid \lambda)}{m !} \sum_{k=1}^{m}\left(\begin{array}{c}
m \\
k
\end{array}\right)(u+v)^{k-1}(-u)^{m-k} \\
& =(\lambda-1) \sum_{m=1}^{\infty} \frac{H_{m}(y-x \mid \lambda)}{m !} \sum_{k=0}^{m-1}(-1)^{m-1-k}\left(\begin{array}{c}
m \\
k+1
\end{array}\right) \sum_{n=0}^{k}\left(\begin{array}{l}
k \\
n
\end{array}\right) u^{m-(n+1)} v^{n} .
\end{aligned}
$$

By changing the order of the summation in the right side of (4.10), we have

$$
M=(\lambda-1) \sum_{n=0}^{\infty} \sum_{m=n+1}^{\infty} \frac{(-1)^{m-1} H_{m}(y-x \mid \lambda)}{m !} u^{m-(n+1)} v^{n} \sum_{k=n}^{m-1}(-1)^{k}\left(\begin{array}{c}
m \\
k+1
\end{array}\right)\left(\begin{array}{l}
k \\
n
\end{array}\right) .
$$

Notice that, for non-negative integer $m, n$ with $m \geq n+1$,

$$
\sum_{k=n}^{m-1}(-1)^{k}\left(\begin{array}{c}
m \\
k+1
\end{array}\right)\left(\begin{array}{l}
k \\
n
\end{array}\right)=(-1)^{n}
$$

by using induction on $m$. Hence, applying (4.12) to (4.11) gives

$$
M=(\lambda-1) \sum_{m=0}^{\infty} \sum_{n=0}^{\infty}(-1)^{m} \frac{m ! \cdot n !}{(m+n+1) !} H_{m+n+1}(y-x \mid \lambda) \frac{u^{m}}{m !} \cdot \frac{v^{n}}{n !} .
$$

By putting (4.13) to (4.6) and then taking $k$ times the derivative with respect to $v$, we get

$$
\begin{aligned}
\sum_{m=0}^{\infty} \sum_{n=0}^{\infty}\left(\sum_{j=0}^{k}\left(\begin{array}{l}
k \\
j
\end{array}\right) \sum_{i=0}^{n}\left(\begin{array}{l}
n \\
i
\end{array}\right) H_{i+j}(y-x \mid \lambda) \beta_{m+n+k-i-j}(x)\right) \frac{u^{m}}{m !} \cdot \frac{v^{n}}{n !} \\
=\sum_{m=0}^{\infty} \sum_{n=0}^{\infty} \frac{1}{\lambda-1} H_{m}\left(x \mid \frac{1}{\lambda}\right) H_{n+k}(y \mid \lambda) \frac{u^{m}}{m !} \cdot \frac{v^{n}}{n !} \\
\quad+\sum_{m=0}^{\infty} \sum_{n=0}^{\infty}\left(\sum_{i=0}^{m}\left(\begin{array}{c}
m \\
i
\end{array}\right)(-1)^{m-i} H_{m-i}(y-x \mid \lambda) \beta_{n+k+i}(y)\right) \frac{u^{m}}{m !} \cdot \frac{v^{n}}{n !} \\
\quad-\sum_{m=0}^{\infty} \sum_{n=0}^{\infty}(-1)^{m} \frac{m ! \cdot(n+k) !}{(m+n+k+1) !} H_{m+n+k+1}(y-x \mid \lambda) \frac{u^{m}}{m !} \cdot \frac{v^{n}}{n !} .
\end{aligned}
$$


Thus, replacing $y$ by $x+y$ and comparing the coefficients of $u^{m} \cdot v^{n} / m ! \cdot n !$ in (4.14) gives Lemma 4.1.

We next give the detailed proof of Theorem 2.4 .

Proof of Theorem 2.4 Obviously, Theorem 2.4 holds trivially when $k=0$ in Lemma 4.1. Now, we assume Theorem 2.4 holds for all positive integers less than $k$. It follows from Lemma 4.1 that, for non-negative integers $m, n$ and positive integer $k$,

$$
\begin{aligned}
\sum_{i=0}^{n}\left(\begin{array}{c}
n \\
i
\end{array}\right) \beta_{m+n-i}(x) H_{k+i}(y \mid \lambda) \\
=\frac{1}{\lambda-1} H_{m}\left(x \mid \frac{1}{\lambda}\right) H_{n+k}(x+y \mid \lambda) \\
\quad+\sum_{i=0}^{m+k}\left(\begin{array}{c}
m \\
i-k
\end{array}\right)(-1)^{m+k-i} H_{m+k-i}(y \mid \lambda) \beta_{n+i}(x+y) \\
\quad-(-1)^{m} \frac{m ! \cdot(n+k) !}{(m+n+k+1) !} H_{m+n+k+1}(y \mid \lambda) \\
\quad-\sum_{j=0}^{k-1}\left(\begin{array}{c}
k \\
j
\end{array}\right) \sum_{i=0}^{n}\left(\begin{array}{c}
n \\
i
\end{array}\right) H_{i+j}(y \mid \lambda) \beta_{m+n+k-i-j}(x) .
\end{aligned}
$$

Since Theorem 2.4 holds for all positive integers less than $k$, we get

$$
\begin{gathered}
\sum_{j=0}^{k-1}\left(\begin{array}{l}
k \\
j
\end{array}\right) \sum_{i=0}^{n}\left(\begin{array}{l}
n \\
i
\end{array}\right) H_{i+j}(y \mid \lambda) \beta_{m+n+k-i-j}(x) \\
=\frac{1}{\lambda-1} \sum_{i=0}^{k-1}(-1)^{i} H_{n+i}(x+y \mid \lambda) H_{m+k-i}\left(x \mid \frac{1}{\lambda}\right) \sum_{j=0}^{k-1}(-1)^{j}\left(\begin{array}{l}
k \\
j
\end{array}\right)\left(\begin{array}{l}
j \\
i
\end{array}\right) \\
+\sum_{i=0}^{m+k}(-1)^{m+k-i} H_{m+k-i}(y \mid \lambda) \beta_{n+i}(x+y) \sum_{j=0}^{k-1}(-1)^{j}\left(\begin{array}{l}
k \\
j
\end{array}\right)\left(\begin{array}{c}
m+k-j \\
i
\end{array}\right) \\
-H_{m+n+k+1}(y \mid \lambda) \frac{1}{n+1} \sum_{j=0}^{k-1}(-1)^{m+k-j} \frac{\left(\begin{array}{c}
k \\
j
\end{array}\right)}{\left(\begin{array}{c}
m+n+k+1-j \\
n+1
\end{array}\right)} .
\end{gathered}
$$

Observe that, for non-negative integers $m, n, k$,

$$
\sum_{j=0}^{k}(-1)^{j} \frac{\left(\begin{array}{c}
k \\
j
\end{array}\right)}{\left(\begin{array}{c}
m+n+j \\
n
\end{array}\right)}=\frac{m ! \cdot(n+k-1) ! \cdot n}{(m+n+k) !}
$$

by using induction on $k$. It follows from (4.17) that, for non-negative integers $m, n$ and positive integer $k$,

$$
\frac{1}{n+1} \sum_{j=0}^{k-1}(-1)^{m+k-j} \frac{\left(\begin{array}{l}
k \\
j
\end{array}\right)}{\left(\begin{array}{c}
m+n+k+1-j \\
n+1
\end{array}\right)}=\frac{(-1)^{m} m ! \cdot(n+k) !}{(m+n+k+1) !}-\frac{(-1)^{m} m ! \cdot n !}{(m+n+1) !} .
$$


Thus, by applying (3.12), (3.13), and (4.18) to (4.16) and combining with (4.15), we get the desired result. This concludes the proof of Theorem 2.4 .

\section{Competing interests}

The authors declare that they have no competing interests.

\section{Authors' contributions}

All authors contributed equally to the writing of this paper. All authors read and approved the final manuscript.

\section{Acknowledgements}

The authors express their gratitude to the anonymous referees for their helpful comments and suggestions in improving this paper. This work is supported by the Foundation for Fostering Talents in Kunming University of Science and Technology (Grant No. KKSY201452090) and the National Natural Science Foundation of P.R. China (Grant No. 51406071, 51666006, 61305057).

Received: 2 September 2016 Accepted: 3 December 2016 Published online: 05 January 2017

\section{References}

1. Frobenius, FG: Über die Bernoullischen Zahlen und die Eulerischen Polynome. Sitzungsber. K. Preußischen Akad. Wissenschaft, Berlin (1910)

2. Araci, S, Acikgoz, M: A note on the Frobenius-Euler numbers and polynomials associated with Bernstein polynomials. Adv. Stud. Contemp. Math. 22, 399-406 (2012)

3. Araci, S, Acikgoz, M: On the von Staudt-Clausen's theorem related to $q$-Frobenius-Euler numbers. J. Number Theory $159,329-339(2016)$

4. Kim, T: Symmetry of power sum polynomials and multivariate fermionic $p$-adic invariant integral on $\mathbb{Z}_{p}$. Russ. J. Math. Phys. 16, 93-96 (2009)

5. Kim, T: An identity of the symmetry for the Frobenius-Euler polynomials associated with the fermionic $p$-adic invariant $q$-integrals on $\mathbb{Z}_{p}$. Rocky Mt. J. Math. 41, 239-247 (2011)

6. Kim, T: Identities involving Frobenius-Euler polynomials arising from non-linear differential equations. J. Number Theory 132, 2854-2865 (2012)

7. Kim, T, Lee, B, Lee, S-H, Rim, S-H: Some identities for the Frobenius-Euler numbers and polynomials. J. Comput. Anal. Appl. 15, 544-551 (2013)

8. Kim, T, Mansour, T: Umbral calculus associated with Frobenius-type Eulerian polynomials. Russ. J. Math. Phys. 21, 484-493 (2014)

9. Simsek, Y, Bayad, A, Lokesha, V: q-Bernstein polynomials related to q-Frobenius-Euler polynomials, I-functions, and q-Stirling numbers. Math. Methods Appl. Sci. 35, 877-884 (2012)

10. Simsek, Y: Generating functions for generalized Stirling type numbers, array type polynomials, Eulerian type polynomials and their applications. Fixed Point Theory Appl. 2013, Article ID 87 (2013)

11. Cohen, H: Number Theory, Volume II, Analytic and Modern Tools. Graduate Texts in Math., vol. 124. Springer, New York (2007)

12. Nielsen, N: Traité élémentaire des nombres de Bernoulli. Gauthier-Villars, Paris (1923)

13. Srivastava, HM, Choi, J: Zeta and q-Zeta Functions and Associated Series and Integrals. Elsevier, Amsterdam (2012)

14. Agoh, T, Dilcher, K: Convolution identities and lacunary recurrences for Bernoulli numbers. J. Number Theory 124 , 105-122 (2007)

15. Agoh, T, Dilcher, K: Reciprocity relations for Bernoulli numbers. Am. Math. Mon. 115, 237-244 (2008)

16. Chen, WYC, Sun, LH: Extended Zeilberger's algorithm for identities on Bernoulli and Euler polynomials. J. Number Theory 129, 2111-2132 (2009)

17. He, Y, Zhang, WP: A convolution formula for Bernoulli polynomials. Ars Comb. 108, 97-104 (2013)

18. He, Y, Zhang, WP: A three-term reciprocity formula for Bernoulli polynomials. Util. Math. 100, $23-31$ (2016)

19. Mu, YP: Linear recurrence relations for sums of products of two terms. Electron. J. Comb. 18, P170 (2011)

20. Yamashita, G: A simple proof of convolution identities of Bernoulli numbers. Proc. Jpn. Acad., Ser. A, Math. Sci. 91, 5-6 (2015)

21. Carlitz, L: The product of two Eulerian polynomials. Math. Mag. 36, 37-41 (1963)

22. Kim, DS, Kim, T: Some new identities of Frobenius-Euler numbers and polynomials. J. Inequal. Appl. 2012, Article ID 307 (2012)

23. He, Y, Wang, SJ: New formulae of products of the Frobenius-Euler polynomials. J. Inequal. Appl. 2014, Article ID 261 (2014)

24. Kim, DS, Kim, T, Lee, S-H, Kim, Y-H: Some identities for the products of two Bernoulli and Euler polynomials. Adv. Differ. Equ. 2012, Article ID 95 (2012)

25. Agoh, T: Convolution identities for Bernoulli and Genocchi polynomials. Electron. J. Comb. 21, Article ID P1.65 (2014)

26. Dunne, GV, Schubert, C: Bernoulli number identities from quantum field theory and topological string theory. Commun. Number Theory Phys. 7, 225-249 (2013)

27. He, Y, Zhang, WP: Some sum relations involving Bernoulli and Euler polynomials. Integral Transforms Spec. Funct. 22, 207-215 (2011)

28. Kim, DS, Kim, T: Identities arising from higher-order Daehee polynomials bases. Open Math. 13, $196-208$ (2015)

29. Pan, H, Sun, Z-W: New identities involving Bernoulli and Euler polynomials. J. Comb. Theory, Ser. A 113, 156-175 (2006)

30. Abramowitz, M, Stegun, IA (eds.): Handbook of Mathematical Functions with Formulas, Graphs, and Mathematical Tables. Applied Mathematics Series, vol. 55. National Bureau of Standards, Washington (1964). Reprinted by Dover Publications, New York, 1965 
31. He, Y, Zhang, WP: Some symmetric identities involving a sequence of polynomials. Electron. J. Comb. 17, Article ID N7 (2010)

32. Kim, DS, Kim, T: A study on the integral of the product of several Bernoulli polynomials. Rocky Mt. J. Math. 44, 1251-1263 (2014)

33. Kim, T, Choi, J: A note on the product of Frobenius-Euler polynomials arising from the $p$-adic integral on $\mathbb{Z}_{p}$. Adv. Stud. Contemp. Math. 22, 215-223 (2012)

34. Kim, T: Some properties on the integral of the product of several Euler polynomials. Quaest. Math. 38, 553-562 (2015)

Submit your manuscript to a SpringerOpen ${ }^{\odot}$ journal and benefit from:

- Convenient online submission

Rigorous peer review

- Immediate publication on acceptance

- Open access: articles freely available online

- High visibility within the field

- Retaining the copyright to your article

Submit your next manuscript at springeropen.com 